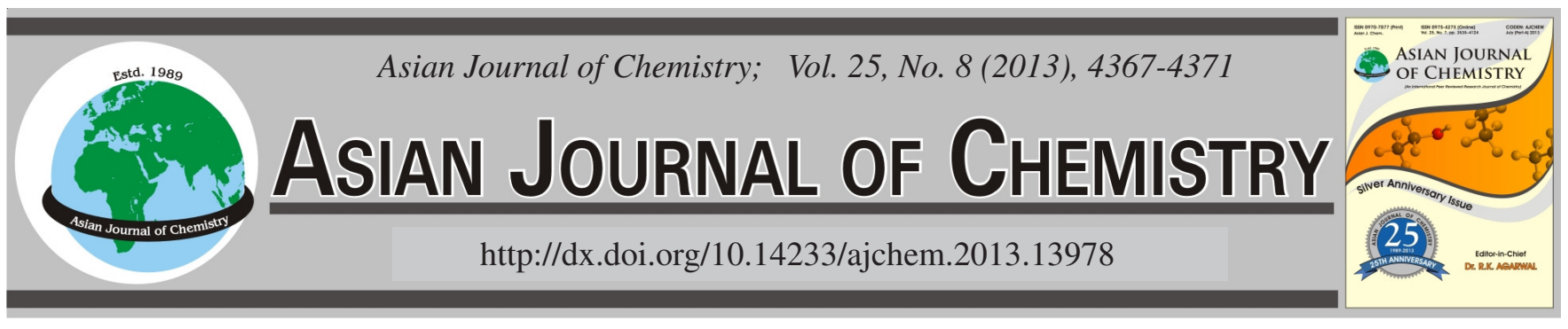

\title{
Analysis of Chemical Constituents of Traditional Chinese \\ Medicinal Formula Huang-Qi-Chi-Feng-Tang by High Performance Liquid Chromatography-Electrospray Ionization-Mass Spectrometry
}

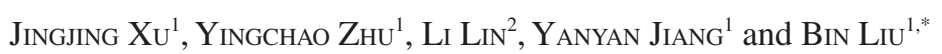

${ }^{1}$ Department of Traditional Chinese Medicine, Beijing University of Chinese Medicine, Beijing, P.R. China

${ }^{2}$ Xiyuan Hospital, China Academy of Chinese Medical Sciences, Beijing, P.R. China

*Corresponding author: Fax: +86 10 84738611; Tel: +86 10 84738629; E-mail: liubinyn67@163.com

\begin{abstract}
Huang-Qi-Chi-Feng-Tang, a three-herb traditional Chinese medicinal formula, has efficient effects on treatment of skin ulcer and pains. However, the active components of this compound Chinese prescription have not been fully explored. In this study, high performance liquid chromatography coupled with electrospray ionization tandem mass spectrometry (HPLC-ESI-MS/MS) was applied for the qualitative analysis of major chemical constituents in Huang-Qi-Chi-Feng-Tang. Samples were separated on a Waters Symmetry Shield $\mathrm{C}_{18}$ column $(150 \mathrm{~mm} \times 4.6 \mathrm{~mm})$ using a gradient system of $20 \%$ methyl alcohol (A)-methyl alcohol (B). The precursor and product ions of analytes were monitored on a hybrid quadrupole linear ion trap mass spectrometer in negative mode. A total of 11 compounds including 3 chromones, 2 monoterpenoids, 5 isoflavone, 1 isoflavane were successfully identified or tentatively characterized based on retention time and MS spectra compared with those of standards or literature data. The results may contribute to the new drug development and illuminate the effective substance basis.

Key Words: Huang-Qi-Chi-Feng-Tang, HPLC-ESI-MS/MS, Qualitative analysis.
\end{abstract}

\section{INTRODUCTION}

Huang-Qi-Chi-Feng-Tang, one of the most popular traditional Chinese medicinal formula comprised of three crude drugs: Milkvetch root (Huang-Qi), Radix paeoniae rubra (Chi-Shao) and Divaricate Saposhnikovia root (Fang-Feng), was firstly recorded on the "Correction on Errors in Medical Classics" (a classical book about Chinese medicine). It has been widely adopted for the clinical use in China for last hundreds of years. According to the literatures ${ }^{1-5}$, this formula mainly applied to treat kinds of skin ulcer and pains was also effective on various diseases, such as chronic nephritis proteinuria, rheumatoid arthritis, cardiovascular and cerebrovascular diseases, epilepsy and even cold. Moreover, recent studies showed that it had antioxidant activity also. In the past few decades, systematic chemical and pharmacological studies have been performed intensively on the three component herbs of HuangQi-Chi-Feng-Tang. The main compositions in its three crude drugs were demonstrated to be of several natural product groups, including saponins, flavoniods, chromones, terpenoids, polysaccharides and phenolics. However, little is known about the chemical constituents of this compound Chinese prescription.
High performance liquid chromatography coupled with electrospray ionization tandem mass spectrometry (HPLCESI-MS/MS) has been widely used in analyzing of multicomponent herbal medicines $^{6-9}$. In this technology, HPLC provided effective chromatographic separation, while MS could efficiently ionize the chemical constituents to produce diagnostic fragment ions by tandem mass spectrometry. HPLCESI-MS/MS facilitates the identification of unknown components in the herbal system remarkably with high sensitivity and accuracy.

In this paper, an HPLC-ESI-MS/MS method was established to identify the major constituents of Huang-Qi-Chi-Feng-Tang for the first time. And 11 compounds were characterized. The result might provide not only the chemical evidence for revealing the material foundation of its therapeutic effects but also the basis for drug metabolism in the new drug exploitation.

\section{EXPERIMENTAL}

HPLC grade methanol (Fisher, USA) was used for HPLC analysis. Ultrapure water was purchased from Hangzhou Wahaha Group Co., Ltd. (Zhejiang, China). Other reagents and chemicals were of analytical grade. 
Reference compounds of 4'-O- $\beta$-glucopyranosyl-5methylvisammionl, cimifugin, ononin, calycosin-7-O- $\beta-D-$ glucoside, prim-O-glucosylcomifugin were prepared by the authors. Paeoniflorin was purchased from the National Institutes for Food and Drug Control (Beijing, China). All the above compounds showed purities of above $98 \%$ by HPLC analysis.

Herbal medicines samples of Milkvetch root, Radix paeoniae rubra and Divaricate Saposhnikovia root were purchased from Anguo Linshi medicinal materials Co., Ltd. in China and were authenticated by Guijun Zhang, Professor of Beijing University of Chinese Medicine.

Standard solutions: Stock solutions were prepared by dissolving appropriate amounts of 6 reference compounds in methanol to make the concentrations of $0.755 \mathrm{mg} / \mathrm{mL}$ for paeoniflorin, $0.094 \mathrm{mg} / \mathrm{mL}$ for prim-O-glucosylcomifugin, $1.096 \mathrm{mg} / \mathrm{mL}$ for calycosin-7-O- $\beta$-D-glucoside, $0.1044 \mathrm{mg} /$ $\mathrm{mL}$ for cimifugin, $0.0828 \mathrm{mg} / \mathrm{mL}$ for 4'-O- $\beta$-glucopyranosyl5-methylvisammionl and $0.78 \mathrm{mg} / \mathrm{mL}$ for ononin. Transfer proper amounts of each stock solution to a $10 \mathrm{~mL}$ volumetric flask and then add methanol to make up the volume to obtain a final mixed standard solution. All the solutions were stored at $4{ }^{\circ} \mathrm{C}$ and brought to room temperature before use.

Sample solution: Milkvetch root was refluxed in proper amount of methanol. The residue following solvent recovery was dissolved in water and purified with a column of macroreticular resin. At first, the resin was washed with water, then washed with methanol. The Huangqi saponin and flavone fraction were obtained by evaporating methanol elution to dryness under reduced pressure with a rotary evaporator. With the same method, Fangfeng chromone fraction and Chishao monoterpene fraction were prepared. According to the formula dosage and the yields, Huang-Qi-Chi-Feng-Tang effective fraction was obtained by grinding and mixing appropriate amount of every fraction.

The Huang-Qi-Chi-Feng-Tang effective fraction $(200 \mathrm{mg}$ ) was then accurately weighed and dissolved in $25 \mathrm{~mL}$ of $75 \%$ ethanol. After $1 \mathrm{~h}$ ultrasonic bath $(500 \mathrm{~W}, 40 \mathrm{kHz})$, the solution was adjusted to the original weight by adding $70 \%$ methanol and filtered through a $0.45 \mu \mathrm{m}$ nylon filter for HPLC analysis.

HPLC-ESI-MS/MS system: HPLC-ESI-MS/MS analysis was performed on an Agilent 1200 series HPLC instrument (Agilent, Waldbronn, Germany) coupled with an API 4000 QTRAP system (Applied Biosystems, Foster City, CA, USA) via an electrospray ionization (ESI) interface. The HPLC instrument was equipped with a binary pump, on-line degasser, auto plate-sampler and column oven. A number of preliminary experiments were performed to optimize mobile phase composition and elution conditions. Finally, analysis was carried out at $35^{\circ} \mathrm{C}$ on a Waters Symmetry Shield $\mathrm{C}_{18}$ $(150 \mathrm{~mm} \times 4.6 \mathrm{~mm})$. The mobile phase system was $20 \%$ methyl alcohol (A)-methyl alcohol (B). A gradient programme was used as follows: 0-30 min, gradient from 80:20 (A:B) to 25:75. The flow rate was kept at $0.8 \mathrm{~mL} / \mathrm{min}$ and the sample volume injected was set at $5 \mu \mathrm{L}$.

The API 4000 QTRAP system is a hybrid triple quadrupole linear ion trap mass spectrometer equipped with Turbo V sources and a Turbo ionspray interface. The analyses were performed using an electrospray ionisation (ESI) source in negative modes. The operating conditions were as follows: the turbo spray temperature was maintained at $550{ }^{\circ} \mathrm{C}$; drying gas $\left(\mathrm{N}_{2}\right)$ flow rate, $10 \mathrm{~L} / \mathrm{min}$; nitrogen was used as for all analyses. The nebulizer gas (Gas 1) and heater gas (Gas 2) were set at 65 and 60 psi, respectively. The curtain gas was kept at 10 psi and interface heater was on. The full-scan mass covered the range from m/z 100-800. All instruments were controlled and data acquisition was carried out using analyst software (version 1.5.1) from Applied Biosystems Sciex.

\section{RESULTS AND DISCUSSION}

Negative ion mode was employed for comprehensive analysis and its total ion current (TIC) chromatograms are shown in Fig. 1. A good separation was achieved within $22 \mathrm{~min}$. Based on MS spectra and retention time $\left(t_{R}\right)$, a total of 11 compounds (Table-1, Fig. 2) were identified or tentatively characterized. Six of them were positively identified by comparing

\begin{tabular}{|c|c|c|c|c|c|c|c|}
\hline \multicolumn{8}{|c|}{$\begin{array}{c}\text { TABLE-1 } \\
\text { HPLC-ESI-MS/MS IDENTIFICATION OF THE COMP }\end{array}$} \\
\hline Peak identification & $\begin{array}{l}\mathrm{RT} \\
(\min )\end{array}$ & $\begin{array}{c}{[\mathrm{M}-\mathrm{H}]^{-}} \\
(\mathrm{m} / \mathrm{z})\end{array}$ & $\begin{array}{l}{\left[\mathrm{M}+\underset{(\mathrm{m} / \mathrm{z})}{\left.\mathrm{CH}_{3} \mathrm{COO}\right]^{-}}\right.} \\
\end{array}$ & $\begin{array}{l}{[\mathrm{M}+\mathrm{HCOO}]^{-}} \\
(\mathrm{m} / \mathrm{z})\end{array}$ & $\begin{array}{l}{[\mathrm{M}+\mathrm{Cl}]^{-}} \\
(\mathrm{m} / \mathrm{z})\end{array}$ & $\begin{array}{l}\mathrm{MS}^{2} \\
(\mathrm{~m} / \mathrm{z})\end{array}$ & $\begin{array}{l}\text { Crude } \\
\text { drug }\end{array}$ \\
\hline 1 Oxypaeoniflorin & 7.98 & 495.3 & & & & $466.7,465.3,327.2$ & 2 \\
\hline 2 Paeoniflorin & 10.26 & 479.3 & 539.1 & 525.1 & 515.1 & $\begin{array}{l}449.2,357.3,339.4,327.2 \\
\quad 309.2,165.1,121.0\end{array}$ & 2 \\
\hline 3 Prim-O-glucosyl-comifugin & 10.53 & 467.3 & 527.1 & 513.2 & 503.3 & $409.4,305.2$ & 3 \\
\hline 4 Calycosin-7-O- $\beta$-D- glucoside & 12.74 & & 505.0 & 491.1 & 481.2 & 283.0 & 1 \\
\hline 5 Cimifugin & 14.29 & 305.1 & & & & $287.2,277.2,259.1,231.0$ & 3 \\
\hline $\begin{array}{l}6 \text { 4'-O-B-Glucopyranosyl-5- } \\
\text { methylvisammionl }\end{array}$ & 15.78 & 451.2 & 511.1 & 497.1 & 487.0 & $271.1,239.0$ & 3 \\
\hline 7 Pratensein-7-O- $\beta$-D-glycoside & 17.25 & 461.2 & 521.2 & 507.1 & 497.1 & $\begin{array}{c}439.8,426.0,299.0,284.5 \\
214.9\end{array}$ & 1 \\
\hline $\begin{array}{l}8 \text { 7-Methoxy-3',5-dihydroxy } \\
\text { isoflavan-4'-O- } \beta \text {-D-glucoside }\end{array}$ & 17.42 & 461.2 & 521.5 & 507.1 & 497.1 & $\begin{array}{l}427.5,365.3,330.2,322.2 \\
299.0,284.2\end{array}$ & 1 \\
\hline 9 Sissotrin & 18.12 & 445.2 & & & & $294.0,283.0$ & 1 \\
\hline 10 Ononin & 18.76 & 429.2 & 489.1 & 475.1 & 465.0 & $394.1,267.1,252.0$ & 1 \\
\hline $\begin{array}{l}11 \text { 2'-Hydroxy-3',4'-dimethoxy } \\
\text { isoflavan-7-O- } \beta \text {-D-glucoside }\end{array}$ & 20.48 & 463.3 & & & & $301.2,286.0$ & 1 \\
\hline
\end{tabular}

${ }^{a}$ The number in crude drug item means which crude drug the ingredient belongs to: (1) Milkvetch root (2) Radix paeoniae rubra (3) Divaricate Saposhnikovia root. 


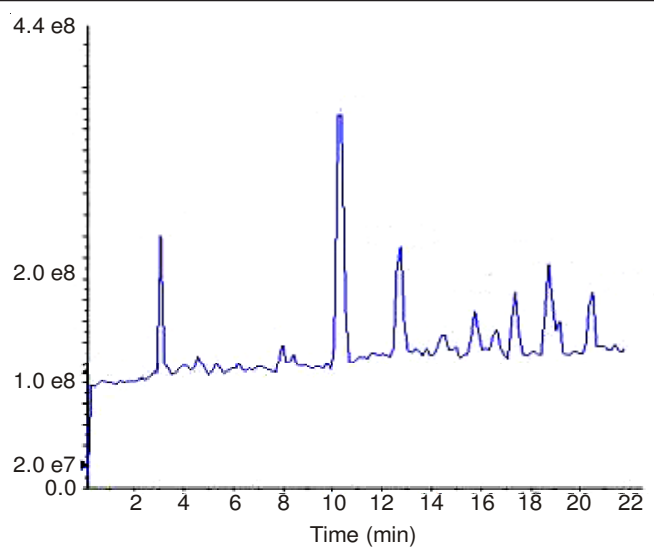

Fig. 1. TIC chromatogram of Huang-Qi-Chi-Feng-Tang in negative ion mode
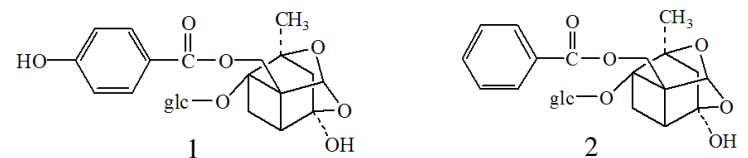<smiles>CCOCc1cc(=O)c2c(OC)c3c(cc2o1)OC(C(C)(C)O)C3</smiles><smiles>CCc1ccc2c(c1)OCC(c1ccc(OC)c(O)c1)C2O</smiles><smiles>COc1c2c(cc3oc(CO)cc(=O)c13)OC(C(C)(C)O)C2</smiles><smiles>COc1c2c(cc3oc(C)cc(=O)c13)OC(C(C)(C)O[Ga])C2</smiles><smiles>[CH]Oc1cc(O)c2c(=O)c(-c3ccc(OC)c(O)c3)coc2c1</smiles><smiles>CCOc1ccc(C2COc3cc(OC)cc(O)c3C2=O)cc1O</smiles><smiles>COc1ccc(-c2coc3cc(OC)cc(O)c3c2=O)cc1</smiles><smiles>COc1ccc(-c2coc3cc(OC)ccc3c2=O)cc1</smiles><smiles></smiles>

Fig. 2. Structures of the 11 compounds in Huang-Qi-Chi-Feng-Tang

the retention time and MS data of the analyzed samples with the respective data obtained from the analysis of reference compounds. The other 5 common peaks were identified by carefully studying on these mass spectrums and comparing with references.

Compounds identified by comparing with reference substances (Fig. 3): Compound 2 with a retention time of $10.26 \mathrm{~min}$ showed $[\mathrm{M}-\mathrm{H}]^{-}$ion at $\mathrm{m} / \mathrm{z} 479.3,\left[\mathrm{M}+\mathrm{CH}_{3} \mathrm{COO}\right]^{-}$ ion at $\mathrm{m} / \mathrm{z} 539.1,\left[\mathrm{M}+\mathrm{HCOO}^{-}\right.$ion at $\mathrm{m} / \mathrm{z} 525.1$ and $[\mathrm{M}+$ $\mathrm{Cl}^{-}$ion at $\mathrm{m} / \mathrm{z} 515.1$, indicating a molecular weight of 480 . The HPLC-MS ${ }^{2}$ spectra of the ion at $\mathrm{m} / \mathrm{z} 479.3$ gave seven major ions at $\mathrm{m} / \mathrm{z} 449.2,357.3,339.4,327.2,309.2,165.1$ and 121.0. By comparison with authentic standard, compound $\mathbf{2}$ was positively identified as paeoniflorin.

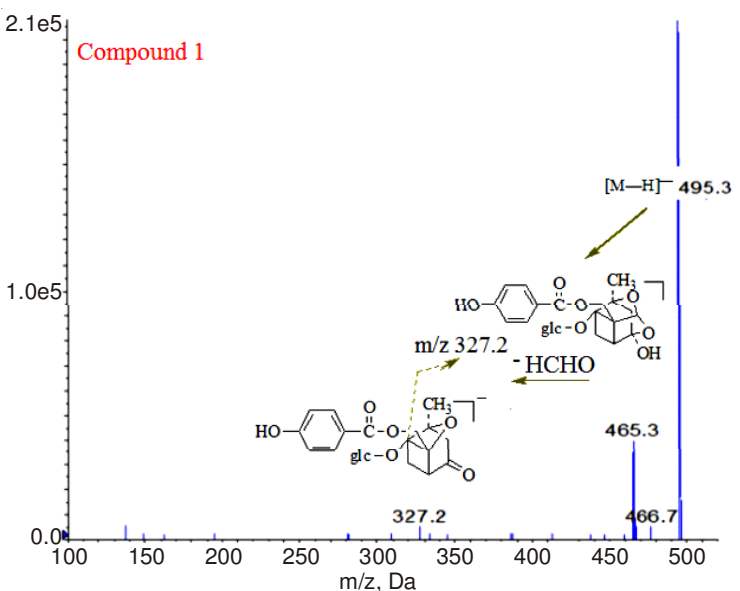

Compound 2
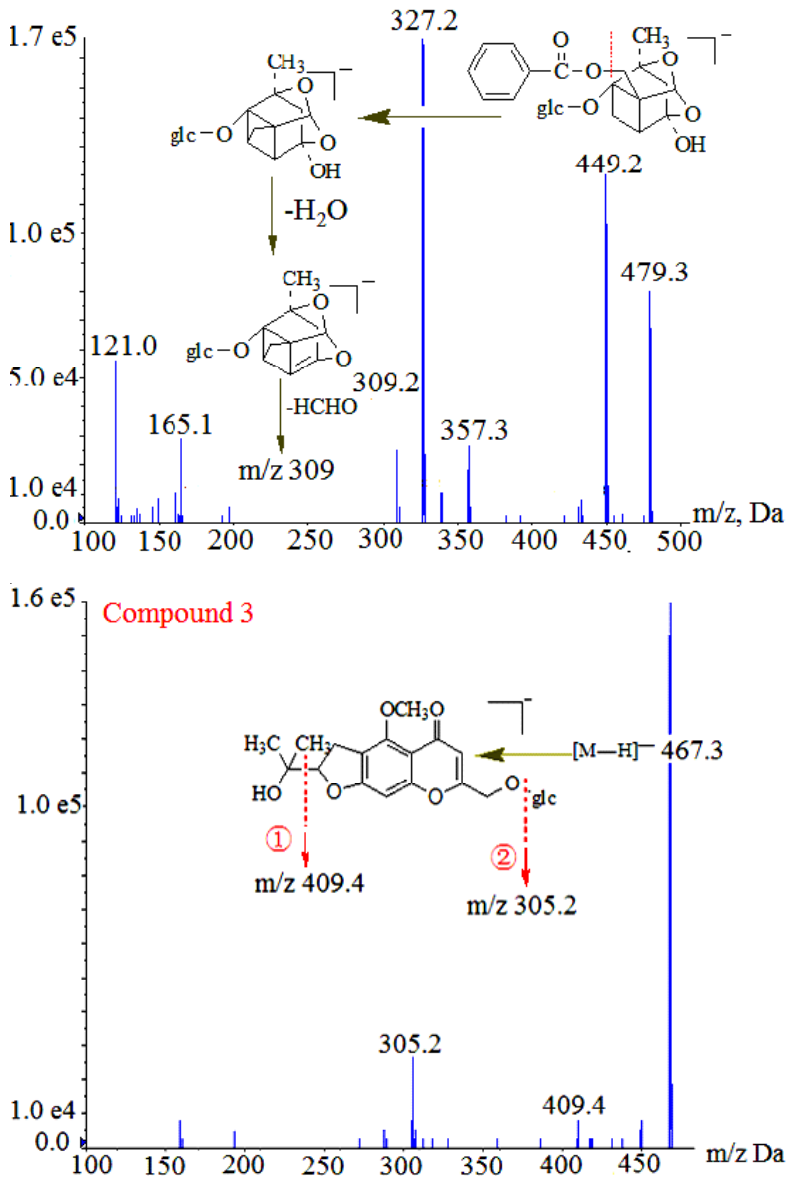

Compound 4

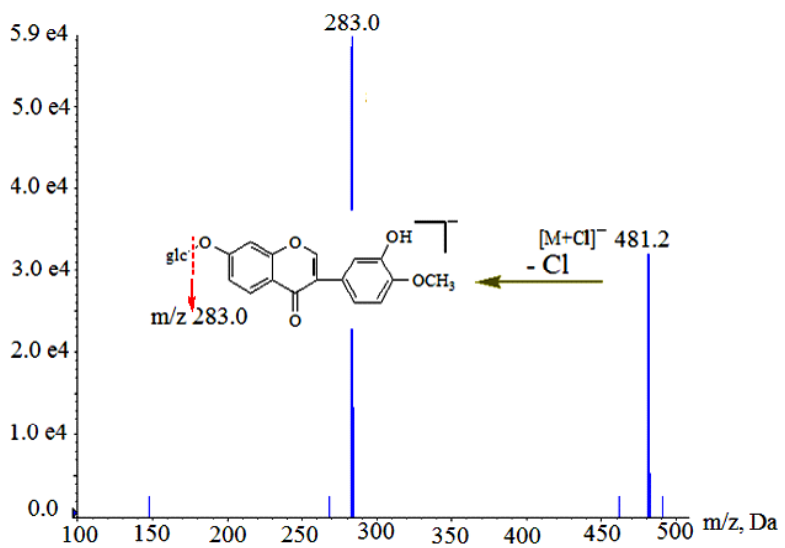




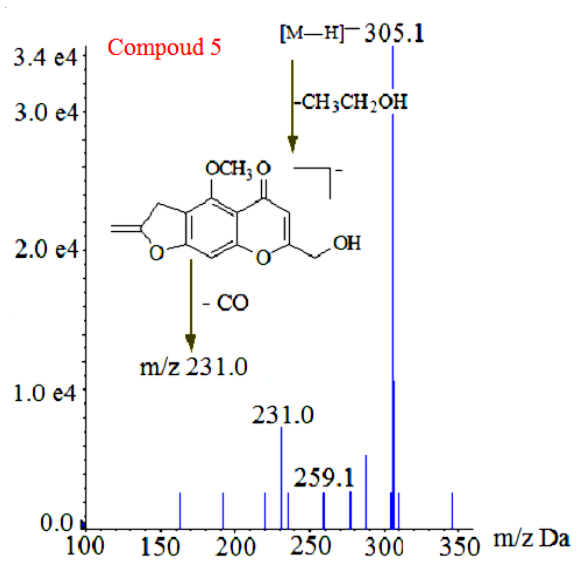

Compound 6
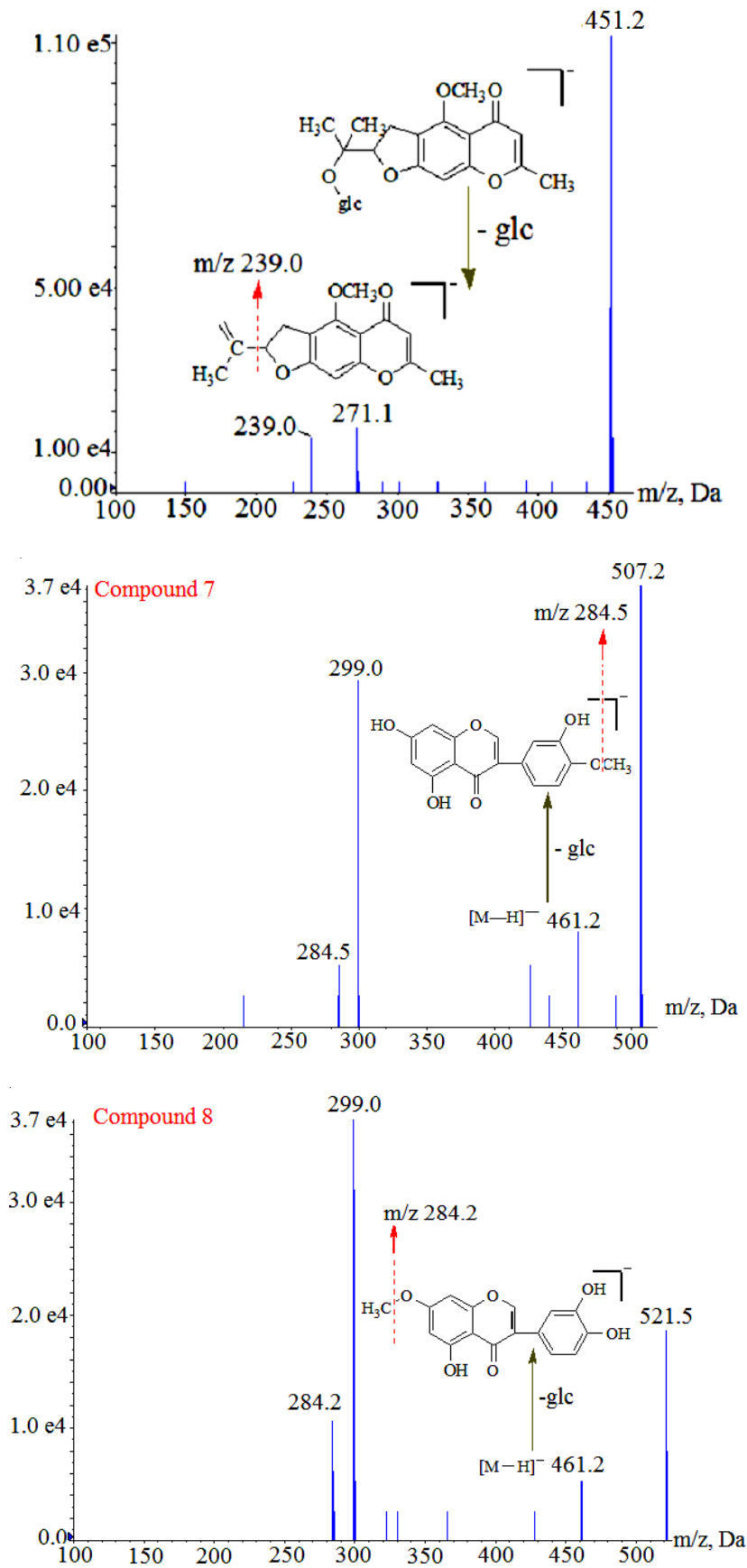
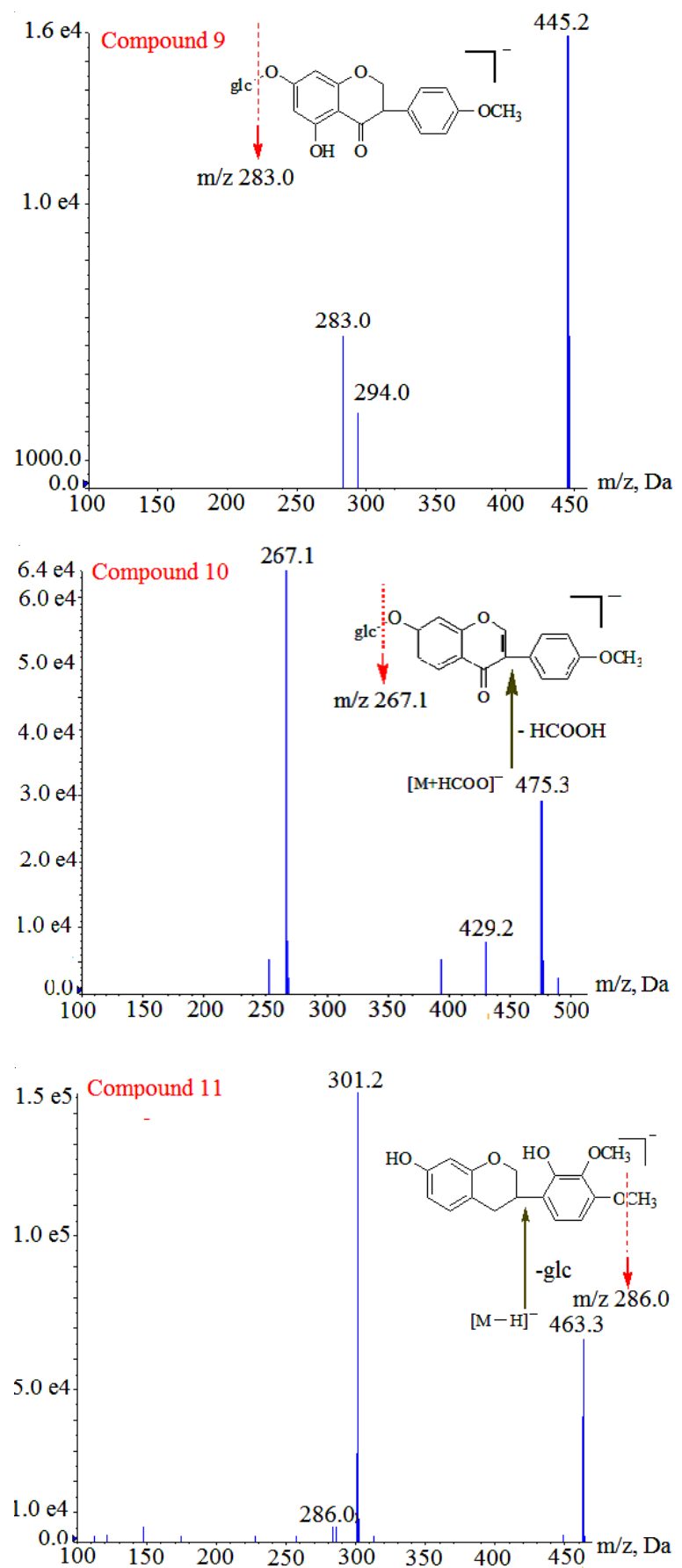

Fig. 3. $\mathrm{MS}^{2}$ spectra of 11 compounds in Huang-Qi-Chi-Feng-Tang. $\mathrm{MS}^{2}$ spectra of oxypaeoniflorin (compound 1), paeoniflorin (compound 2), prim-O-glucosylcomifugin (compound 3 ), calycosin-7-O- $\beta$-Dglucoside (compound 4), acimifugin (compound 5), 4'-O- $\beta$ glucopyranosyl-5-methylvisammionl (compound 6), pratensein-7O-B-D-glucoside (compound 7), 7-methoxy-3',5-dihydroxyisoflavan4'-O- $\beta$-D-glucoside (compound 8 ), sissotrin (compound 9), ononin (compound 10), 2'-hydroxy-3',4'-dimethoxyisoflavan-7-O- $\beta$-Dglucoside (compound 11)

Compound 3 with a retention time of 10.53 min produced $476.3[\mathrm{M}-\mathrm{H}]^{-}, 527.1\left[\mathrm{M}+\mathrm{CH}_{3} \mathrm{COO}^{-}, 513.2\left[\mathrm{M}+\mathrm{HCOO}^{-}\right.\right.$ and 503. $3[\mathrm{M}+\mathrm{Cl}]^{-}$, explaining its molecular weight was 474. The HPLC-MS ${ }^{2}$ spectra of the ion at $\mathrm{m} / \mathrm{z} 476.3$ showed two major ions at $\mathrm{m} / \mathrm{z} 409.4$ and 305.2. Compared with authentic standard, compound $\mathbf{3}$ was identified as prim-Oglucosylcomifugin. 
The retention time of compound $\mathbf{4}$ was $12.74 \mathrm{~min}$ and its quasi-molecular ions were $505.0\left[\mathrm{M}+\mathrm{CH}_{3} \mathrm{COO}^{-}, 491.1[\mathrm{M}\right.$ $+\mathrm{HCOO}^{-}$and $481.2[\mathrm{M}+\mathrm{Cl}]^{-}$. Further investigation in the HPLC-MS ${ }^{2}$ experiment of the $\mathrm{m} / \mathrm{z} 481.2$ ion yielded a fragment ion at $\mathrm{m} / \mathrm{z}$ 283.0. Compound 4 was identified as calycosin-7O- $\beta$-D-glucoside by comparing with authentic compound.

The retention time of compound 5 was $14.29 \mathrm{~min}$. It produced the $[\mathrm{M}-\mathrm{H}]^{-}$ion at $\mathrm{m} / \mathrm{z} 305.1$ and further yielded the fragment ions at $\mathrm{m} / \mathrm{z} 287.2,277.2,259.1,231.0$, revealing a molecular mass of 306. Compared with authentic standard, compound $\mathbf{5}$ was identified as cimifugin.

To compound 6, $511.1\left[\mathrm{M}+\mathrm{CH}_{3} \mathrm{COO}\right]^{-}, 497.1\left[\mathrm{M}+\mathrm{HCOO}^{-}\right.$ , $487.0[\mathrm{M}+\mathrm{Cl}]^{-}$and $451.2[\mathrm{M}-\mathrm{H}]^{-}$were detected, suggesting a molecular mass of 452 . The fragment ions observed at $\mathrm{m} / \mathrm{z}$ 271.1, 239.0 in the HPLC-MS ${ }^{2}$ spectrum came from the ion at $\mathrm{m} / \mathrm{z}$ 451.2. Its retention time was $15.78 \mathrm{~min}$. By comparison with authentic compound, compound 6 was identified as 4'O- $\beta$-glucopyranosyl-5-methylvisammionl.

Similarly, the molecular mass of compound $\mathbf{1 0}$ was determined as 430 from its quasi-molecular ions: [M-H] $]^{-}(\mathrm{m} / \mathrm{z}$ 429.2), $\left[\mathrm{M}+\mathrm{CH}_{3} \mathrm{COO}\right]^{-}(\mathrm{m} / \mathrm{z} 489.1),\left[\mathrm{M}+\mathrm{HCOO}^{-}(\mathrm{m} / \mathrm{z}\right.$ 475.1), $[\mathrm{M}+\mathrm{Cl}]^{-}\left(\mathrm{m} / \mathrm{z}\right.$ 465.0). During the HPLC-MS ${ }^{2}$ experiment, the ion at $\mathrm{m} / \mathrm{z} 429.2$ formed three fragment ions at $\mathrm{m} / \mathrm{z}$ $394.1,267.1,252.0$, respectively. Its retention time (18.76 min) and mass datas were in complete accord with the reference compound of ononin. So compound $\mathbf{1 0}$ was identified as ononin.

Compounds identified by comparing with references: Compound 1 with a retention time of 7.98 min gave a ion peak at $\mathrm{m} / \mathrm{z} 495.3$ in negative mode. Its product ions at $\mathrm{m} / \mathrm{z}$ 466.7, 465.3, 327.2 were observed in the HPLC-MS ${ }^{2}$ spectrum. By referring to literature data ${ }^{9}$, compound $\mathbf{1}$ was tentatively identified as oxypaeoniflorin.

Compound $\mathbf{7}$ and $\mathbf{8}$ were a pair of isomers. Both of them gave a $[\mathrm{M}-\mathrm{H}]-$ ion at $\mathrm{m} / \mathrm{z}$ 461.2. Their $\mathrm{MS}^{2}$ spectrum gave the ion at $\mathrm{m} / \mathrm{z} 299.0$, involving the loss of a glucosyl group, then $\mathrm{m} / \mathrm{z} 299.0$ yielded ion at $\mathrm{m} / \mathrm{z} 284.5$, suggesting the presence of a methyl group. By referring to literature data ${ }^{10}$, there is a isoflavone, named pratensein-7-O- $\beta$-D-glucoside, consistent with the above data. Since there is no report in the literature that 7-methoxy-3', 5-dihydroxyisoflavan-4'-O- $\beta$-D-glucoside exists in the three component herbs of this formula, the identification of 7-methoxy-3', 5-dihydroxyisoflavan-4'-O- $\beta$ $\mathrm{D}$-glucoside is still tentative. Moreover, we could also not prove which peak the compound belongs to.

Compound 9 with a retention time of 18.12 min exhibited its quasi-molecular ion at $\mathrm{m} / \mathrm{z} 445.2[\mathrm{M}-\mathrm{H}]^{-}$. By the loss of a glucose group, the precursor ion $[\mathrm{M}-\mathrm{H}]^{-}(\mathrm{m} / \mathrm{z}$ 445.2) showed peak at $\mathrm{m} / \mathrm{z} 283$. Based on the reference data ${ }^{11,12}$, compound 9 was tentatively identified as sissotrin.

Compound 11 with a retention time of 20.48 min showed $[\mathrm{M}-\mathrm{H}]^{-}$ion at 463.3. And its $\mathrm{MS}^{2}$ spectrum produced ions at $\mathrm{m} / \mathrm{z} 301.2$ and 286.0, resulted from the successive loss of glucosyl group and methyl group. Combining literature data ${ }^{13-15}$, we tentatively identified compound $\mathbf{1 1}$ as 2'-hydroxy-3',4'dimethoxyisoflavan-7-O- $\beta$-D-glucoside.

\section{Conclusion}

In this study, an efficient and accurate method using HPLC-ESI-MS/MS was established for chemical investigation on Huang-Qi-Chi-Feng-Tang. To the best of our knowledge this is the first report on the analysis of this effective formula. According to the retention time and MS data, 11 compounds including 3 chromones, 2 monoterpenoids, 5 isoflavone, 1 isoflavane were unambiguously identified or tentatively characterized by comparison with the standard substance and the literature data. On the basis of illuminating the effective substance foundation, the present study could not only provide comprehensive information for clinical applications, but also promote pharmacological research and new drug exploitation. Moreover, our present method could simplify the tedious conventional phytochemical separation procedures of HuangQi-Chi-Feng-Tang.

\section{REFERENCES}

1. H. Ma, Q. Zhu, X.Y. Sun and X.D. Li, Shi Zhen J. Trad. Chin. Med. Res., 9, 324 (1998).

2. Z.G. Li, Jilin J. Trad. Chin. Med., 26, 33 (2006).

3. L.H. Zhou and C.Z. Liu, Jiangxi J. Trad. Chin. Med., 35, 39 (2004).

4. H.X. Liu and C.P. Yin, Chin. J. Integr. Med. Cardio-/Cerebrovascular Dis., 6, 27 (2008).

5. C.A. Yuan, Hunan J. Trad. Chin. Med., 10, 18 (1994).

6. H.J. Zhang, P. Shen and Y.Y. Cheng, J. Pharm. Biomed. Anal., 34, 705 (2004).

7. Zh. X. Yan, X.H. Yang, J.B. Wu, H. Su, CH. Chen and Y. Chen, Anal. Chim. Acta, 691, 110 (2011).

8. J. Han, M. Ye, H. Guo, M. Yang, B.R. Wang and D.A. Guo, J. Pharm. Biomed. Anal., 44, 430 (2007).

9. S.J. Xu, L. Yang, M. Zhang and Z.T. Wang, Acta Pharm. Sin., 41, 852 (2006).

10. X. Zhang, H.B. Xiao, X.Y. Xue, Y.G. Sun and X.M. Liang, J. Sep. Sci., 30, 2059 (2007).

11. Q. Ma, J.L. Zhang, Y.X. Zhou, H.M. Lei and C.H. Wang, Chin. J. Anal. Chem., 34, 247 (2006).

12. X.G. He, L.Z. Lin and L.Z. Lian, J. Chromatogr. A, 75, 127 (1996).

13. L.W. Qi, Q.T. Yu, P. Li, S.L. Li, Y.X. Wang, L.H. Sheng and L. Yi, J. Chromatogr. A, 1134, 162 (2006).

14. L.W. Qi, P. Li, S.L. Li, L.H. Sheng, R.Y. Li, Y. Song and H.J. Li, J. Sep. Sci., 29, 2211 (2006).

15. L.W. Qi, J. Cao, P. Li, Q.T. Yu, X.D. Wen, Y.X. Wang, Ch. Y. Li, K.D. Bao, X.X. Ge and X.L. Cheng, J. Chromatogr. A, 1203, 27 (2008). 